\title{
Dietary intake of marine polyunsaturated n-3 fatty acids and risk of recurrent venous thromboembolism
}

Running head: Omega-3 fatty acids and recurrent venous thromboembolism

Trond Isaksen*†, Line H. Evensen*†, Sigrid K. Brækkan*†, John-Bjarne Hansen*†

*K.G Jebsen Thrombosis Research and Expertise Center (TREC), Department of Clinical

Medicine, UiT-The Arctic University of Norway, Troms $\varnothing$, Norway

$\dagger$ Division of Internal Medicine, University Hospital of North Norway, Troms $\emptyset$, Norway

\section{Correspondence to:}

Trond Isaksen

K.G. Jebsen Thrombosis Research and Expertise Center (TREC)

Department of Clinical Medicine, UiT-The Arctic University of Norway

9037 Troms $\varnothing$, Norway

E-mail: trond.isaksen@uit.no

Telephone: +4795189233

Word count abstract: 238

Word count text: 3978 


\begin{abstract}
Background: Limited knowledge exists on the association between intake of long-chained n-3 polyunsaturated fatty acids (n-3 PUFAs) and risk of recurrence and all-cause mortality in patients with venous thromboembolism (VTE).
\end{abstract}

Objectives: To investigate whether intake of marine n-3 PUFAs was associated with risk of recurrence and mortality in patients with incident VTE.

Methods: A total of 595 patients with incident VTE and available data on n-3 PUFA intake were derived from the Troms $\varnothing$ Study surveys 4 (1994-95) and 6 (2007-08). Weekly intake of n-3 PUFAs was categorized as low, medium and high based on tertiles. Recurrent VTEs and all-cause mortality were registered up to December 31, 2016. Hazard ratios (HRs) were calculated using Cox-regression models with the low intake-category as reference.

Results: There were 98 recurrent VTEs and 227 deaths during follow-up. Overall, we found no association between intake of n-3 PUFAs and risk of recurrent VTE. However, inverse associations were found for high intakes in patients with unprovoked VTE (HR 0.45, 95\% CI 0.20-1.01), cancer-free patients (HR 0.51, 95\% CI: 0.27-0.95), and DVT patients (HR 0.49, 95\% CI: 0.24-0.97). The inverse associations were more evident when follow-up was restricted to the time after discontinuation of anticoagulant therapy. No association was observed between intake of n-3 PUFAs and mortality after incident VTE.

Conclusions: A high dietary intake of marine n-3 PUFAs was associated with lower risk of recurrent VTE after unprovoked index events, DVT and in cancer-free patients. 
Keywords: Diet - Omega-3 fatty acids - Recurrence - Risk factors - Venous

thromboembolism 


\section{INTRODUCTION}

Venous thromboembolism (VTE), including deep vein thrombosis (DVT) and pulmonary embolism (PE), is a common and burdensome disease associated with serious consequences $(1,2)$. The one- and eight-year mortality after VTE is $22-36 \%$ and $30-52 \%$, respectively, with the highest rates observed after acute PE (3-6). Notably, patients with VTE have an elevated risk of mortality for up to three decades after the incident event when compared to the general population (7). Recurrent events are common, especially during the first year after the incident event, and the ten-year cumulative incidence of recurrence is $30-40 \%$ (4-6). The risk of recurrence is dependent on the etiology of the index event. The lowest risk is observed in patients with VTE provoked by a major transient risk factor (e.g., major surgery), an intermediate risk is observed in patients where a provoking factor cannot be identified, and the highest risk is recognized in presence of a major persistent risk factor (e.g., cancer) (8). Although anticoagulant therapy efficiently prevents VTE recurrence, such treatment is most often time-limited as the benefit must be balanced against the risk of bleeding $(9,10)$. In order to improve strategies for secondary prevention, there is a need to identify life-style factors that are inversely associated with adverse outcomes after VTE.

Fish and marine food products are advocated as important determinants of a healthy diet, and the long-chained n-3 polyunsaturated fatty acids (n-3 PUFAs; eicosapentaenoic acid (EPA), docosapentaenoic acid (DPA) and docosahexaenoic acid (DHA)) are identified as key bioactive compounds (11-13). n-3 PUFAs are reported to be associated with downregulation of inflammation (14), platelet function (15), platelet endothelium-interactions $(16,17)$ and tissue factor expression (18), which are key pathways in the VTE pathogenesis. We and others have previously suggested a favorable association between dietary intake of fish and marine food products, particularly those high in n-3 PUFAs, and the risk of incident VTE (1922). Specifically, in our recent report based on the Troms $\emptyset$ Study, we found that moderate and 
high intake of marine n-3 PUFA was associated with 22-26\% lower risk of VTE (22). However, the role of n-3 PUFAs in relation to complications following VTE, such as recurrence and mortality, is largely unknown.

To our knowledge, only one study has investigated the association between n-3 PUFAs and risk of recurrence and mortality after VTE. In the Swiss Cohort of Elderly Patients with Venous Thromboembolism (SWITCO65+), n-3 PUFA (marine- and plantbased) levels in the erythrocyte membrane were measured in VTE patients aged $\geq 65$ years, and the six-month and three-year risks of recurrence and mortality were investigated (23). They found that medium or high content of n-3 PUFAs in the erythrocyte membrane was associated with a lower six-month recurrence risk, and a lower risk of mortality at both time points. However, although, the fatty acid composition in the erythrocyte membrane reflects the fatty acid composition in the diet $(24,25)$, the association between dietary intake of $n-3$ PUFAs and risk of recurrent VTE has not been studied. Further, whether the reported associations apply to VTE patients with a wider age-range and in a longer time-perspective, is not known. Finally, the plant-based and the marine-derived n-3 PUFAs may not be equally important in this context. Therefore, the aims of the present study was to investigate the association between dietary intake of marine n-3 PUFAs and the risk of recurrence and allcause mortality in VTE patients recruited from a population-based cohort with long followup. 


\section{METHODS}

\section{Study population}

The Troms $\varnothing$ Study is a population-based single-center cohort study with repeated health surveys of inhabitants of the Troms $\varnothing$ municipality in Norway (26). The study was approved by the Regional Committee for Medical and Health Research Ethics, and all participants provided written informed consent prior to inclusion. Patients with incident VTE were recruited among participants in the fourth (1994-95) and sixth (2007-08) surveys of the Troms $\varnothing$ study. The source population comprised of 29,538 unique individuals, of which 10,304 took part in both surveys. The participants were followed from study inclusion to the end of the study period (December 31, 2016). All incident VTE events were identified and adjudicated by trained personnel searching the hospital discharge registry, the radiology procedure registry and the autopsy registry at the University Hospital of North Norway (UNN), as previously described in detail (27). UNN is the only hospital and the exclusive provider of relevant diagnostic radiology and hospital care in the study region, and manages essentially all in- and outpatients in Troms $\emptyset$ with a VTE diagnosis. Adjudication criteria for VTE were (i) signs and symptoms DVT or PE, (ii) objective confirmation by radiological procedures (compression ultrasonography, venography, spiral computed tomography, perfusion-ventilation scan or pulmonary angiography) or autopsy (only including cases where VTE was a significant contributor, or cause of death), (iii) a diagnosis of DVT or PE in the patient journal, and (iv) treatment initiation unless contraindications were specified.

There were of 896 incident VTE events during follow-up, and these formed the basis of our study population. Participants $(n=301)$ with incomplete information on $n-3$ PUFA intake were excluded, and 595 patients with incident VTE were included in the present study. A comparison of included VTE patients and those excluded due to incomplete data on fish intake and use of fish oil supplements is shown in Table S1. 
All VTE events were classified as provoked or unprovoked based on the presence of provoking factors at the time of diagnosis. Provoking factors were major surgery or trauma (within 8 weeks prior to the event), acute medical condition (acute myocardial infarction, acute ischemic stroke, major infectious disease), cancer, immobilization (bed rest $\geq 3$ days or confinement to wheelchair, long distance travel $\geq 4 \mathrm{~h}$. within the last 14 days) or a factor specifically described as provoking in the medical record, e.g. intravascular catheter. A VTE was classified as unprovoked if no provoking factor was reported.

\section{Characteristics}

Information on anthropometry, dietary habits, physical activity and medical history was obtained from physical examinations and self-administered questionnaires at inclusion in Troms $\varnothing 4$ or 6 . Height, weight and blood pressure were measured by standardized procedures, as described elsewhere (27). Body mass index (BMI) was calculated as weight in kilograms divided by the square of height in meters $\left(\mathrm{kg} / \mathrm{m}^{2}\right)$. History of cardiovascular disease (CVD), diabetes, alcohol intake, physical activity, education level and smoking habits were reported via questionnaires. Information on cancer was obtained from the Cancer Registry of Norway. Active cancer was recorded when a cancer diagnosis was made in the time period from two years before to one year after the incident VTE event. Information on preplanned duration of anticoagulant treatment was obtained from the patients' medical records.

\section{Assessment of marine n-3 PUFA intake}

The assessment of marine n-3 PUFA intake in the Troms $\varnothing$ Study has been described in detail previously $(22,28)$. In brief, all participants in Troms $\varnothing 4$ and 6 were asked to complete food 
frequency questionnaires (FFQs), which included questions about how frequently they had fat fish and lean fish for dinner, how frequently they had fish as bread spread, and how often they used fish-oil supplements. In order to estimate the total intake of n-3 PUFAs, we first calculated the average content of n-3 PUFAs in different food items and supplements based on information obtained from official web resources $(11,29,30)$, as previously described in detail (22). One serving unit of fish for dinner was defined as $200 \mathrm{~g}$, and one serving unit of fish as bread spread was defined as $25 \mathrm{~g}$. Total weekly intake of $\mathrm{n}-3$ PUFAs was calculated by summarizing the amount of n-3 PUFAs derived from a person's weekly intake of fat and lean fish, fish as bread spread and fish oil supplements. For participants with data on n-3 PUFAs intake in both Troms $\varnothing 4$ and 6, the data with shortest proximity to the incident VTE event (before or after) was used, as it was assumed to be the most relevant intake. For the remaining, the available data, either from Troms $\varnothing 4$ or 6 , was used. Eventually, there were 342 and 253 participants with data form Troms $\varnothing 4$ and 6, respectively.

\section{Outcome assessment}

All recurrent VTE events during follow-up were identified and adjudicated using the same procedure as described for the incident events. Data on mortality was obtained from the Norwegian Population Registry.

\section{Statistical analysis}

Person-years of follow were accrued from the date of the incident VTE to the date of recurrent VTE, death, migration or the end of the study period (December 31, 2016), whichever occurred first. Recurrent VTE was not included as a censoring event in the analyses on mortality. The study population was categorized into tertiles according to the 
estimated intake of n-3 PUFAs. The ranges were $<8.19,8.19-29.1$ and $>29.1 \mathrm{~g} /$ week for low, medium and high intake, respectively.

Crude incidence rates of VTE recurrence (IRs) and mortality rates (MRs) according to n-3 PUFA intake were calculated and expressed as number of events per 100 person-years with $95 \%$ confidence intervals (CIs). Cox proportional hazards regression models were used to estimate hazard ratios (HRs) with $95 \%$ CIs for recurrence and mortality with the lowest tertile of n-3 PUFA intake as the reference. Calendar time was used as time scale for the recurrence analyses, and attained age was used as time scale in the mortality analyses. The analyses were performed in two models for VTE recurrence and two models for mortality. For recurrence, model 1 was unadjusted and model 2 was adjusted for age, sex and BMI. In addition, to account for death as a competing event to VTE recurrence, sub-distribution hazard ratios (SHR) were calculated according to the method by Fine and Gray (31). For mortality, model 1 included age (as time scale), while model 2 additionally included sex, BMI, systolic blood pressure and smoking. For both outcomes, sensitivity analyses excluding participants with active cancer at the time of incident VTE, were performed. Moreover, we performed sensitivity analysis where follow-up started at the time of discontinuation of anticoagulant treatment. IRs/MRs and HRs for recurrence and mortality according to n-3 PUFA intake were calculated overall, and in subgroups stratified by characteristics of the incident event (unprovoked and provoked, DVT and PE). The proportional hazards assumption was evaluated and verified on the basis of Schoenfeld residuals. The cumulative incidence of VTE recurrence according to n-3 PUFA intake was visualized in 1-Kaplan-Meier plots. Statistical analyses were performed with STATA version 15.1 (Stata Corp, College Station, TX, USA) and R version 3.3.3 (The R Foundation for Statistical Computing, Vienna, Austria). 


\section{RESULTS}

Characteristics of first and recurrent VTE according to categories of n-3 PUFAs intake are summarized in Table 1. Overall, the mean age at incident VTE was $67( \pm 13)$ years and 51\% were men. Further, $38 \%$ of the incident VTE events were unprovoked and 58\% presented as a DVT. The age at incident VTE increased with increasing intake of n-3 PUFAs. Likewise, the prevalence of CVD increased across tertiles, while the prevalence of cancer was lowest in the middle tertile. The mean age at recurrent VTE was $69( \pm 12)$ years, and $61 \%$ were men.

\section{Recurrence}

Among the 595 patients with incident VTE, there were 98 recurrences during a median follow-up of 3.6 years. HRs of recurrence according to n-3 PUFA intake are shown in Table 2. Overall, there was no significant association between n-3 PUFA intake and risk of recurrence. Compared to the lowest tertile, the HR for tertile 2 was 1.09 (95\% CI: 0.69-1.74) and the HR for tertile 3 was 0.77 (95\% CI: 0.45-1.30) in the multivariable model (model 2). However, analyses stratified by characteristics of the incident event showed that a high n-3 PUFA intake (i.e. tertile 3) was associated with a 55\% lower recurrence risk after unprovoked VTE (HR model 2: 0.45, 95\% CI 0.20-1.01), and a 51\% lower risk after DVT (HR model 2: 0.49, 95\% CI 0.24-0.97). Further adjustment for alcohol consumption and physical activity did not affect our results (data not shown).

The ten-year cumulative incidence of recurrence after VTE according to tertiles of n-3 PUFA intake is shown in Fig. 1 and 2. The beneficial effect of a high n-3 PUFA intake on recurrence risk in patients with unprovoked VTE and DVT appeared to occur approximately two years after the incident event. 
Sensitivity analyses excluding participants with active cancer $(n=100)$ at the time of incident VTE are shown in Table 3. The results were comparable to the main analyses, but the inverse associations between n-3 PUFA intake and recurrence risk were stronger. Specifically, a high intake of n-3 PUFAs (tertile 3) was associated with a significant $49 \%$ lower risk of recurrence (HR model 2: 0.51, 95\% CI: 0.27-0.95), which was most pronounced in those with unprovoked VTE (HR model 2: 0.41, 95\% CI: 0.17-1.03) and DVT (HR model 2: 0.31, 95\% CI: 0.13-0.71). However, in contrast to the main analyses, a high n-3 PUFA intake was also associated with a non-significant lower risk of recurrence after provoked VTE (HR: 0.66, 95\% CI: 0.27-1.57). We observed stronger inverse associations when start of follow-up was restricted to the period after discontinuation of anticoagulant treatment (Table S2). For high n3 PUFA intake we observed significantly reduced risk of recurrence after overall VTE (HR model 2: 0.42, 95\% CI: 0.21-0.82), unprovoked VTE (HR model 2: 0.31, 95\% CI: 0.12-0.83), and after DVT (HR model 2: 0.30, 95\% CI: 0.13-0.73). Additionally, we observed a nonsignificant reduced recurrence risk after provoked VTE and after PE in this analysis (HR model 2: 0.61, 95\% CI: 0.24-1.55, and HR: 0.70, 95\% CI: 0.24-2.04, respectively). In analyses taking competing risk by death into account, the SHRs were generally comparable to the HRs obtained from the Cox regression models (Tables 2 and 3).

\section{All-cause mortality}

There were 227 deaths during a median follow-up of 4.5 years. HRs of all-cause mortality according to n-3 PUFA intake are shown in Table 4. Overall, there was no association between intake of n-3 PUFAs and the risk of mortality after incident VTE in the multivariable model (HR tertile 2: 0.96, 95\% CI: 0.69-1.35; HR tertile 3: 1.02, 95\% CI: 0.73-1.43). Similarly, no association was observed between n-3 PUFA intake and mortality risk in analyses stratified by characteristics of the incident event. The results from the sensitivity 
analyses restricted to participants without active cancer were generally comparable to the results from the main analyses (Table 5). However, a non-significant lower mortality risk was observed after provoked VTE in those with a high n-3 PUFA intake (HR model 2: 0.68, 95\% CI: 0.39-1.20).

\section{DISCUSSION}

In the present study, we investigated the association between dietary intake of marine n-3 PUFAs and the risk of VTE recurrence and all-cause mortality in patients with incident VTE recruited from a general population. We found that a high intake of n-3 PUFAs (upper tertile, $>29.1 \mathrm{~g} /$ week) was associated with a lower recurrence risk compared to a low intake (lower tertile, $<8.2 \mathrm{~g} /$ week) in patients with unprovoked incident events and in those with DVT. In contrast, there was no association between n-3 PUFAs intake and risk of mortality after incident VTE. The confidence intervals were wide, particularly in some subgroups, and our findings should therefore be interpreted with caution.

We and others have previously reported on the association between fish or n-3 PUFA intake and the risk of incident VTE. Although findings have been inconsistent, the available data suggests that intake of n-3 PUFAs may lower the risk of incident VTE (19-21). In our previous report, we found an inverse association between intake of marine n-3 PUFAs and risk of VTE, while there was no association between total fish intake and VTE risk (22). Marine n-3 PUFAs have an impact on several molecular pathways and risk factors of relevance in the VTE pathogenesis. These include downregulation of inflammation (14), tissue-factor expression $(18,32)$, platelet function (15), platelet endothelium-interactions (16, 17), and possibly hepatic excretion of coagulation factors (33). Moreover, n-3 PUFAs alternate cell membrane permeability and functionality, and have antiarrhythmic properties 
To our knowledge, our study is among the first to explore the association between marine n-3 PUFAs and the risk of recurrence and mortality in patients with incident VTE. Reiner and colleagues investigated the association between levels n-3 PUFAs in the erythrocyte membrane, and the risk of recurrence and mortality in elderly VTE patients (23). Although there were few recurrent events $(n=22)$, they found that a moderate or high level of n-3 PUFAs was associated with a 61-83\% lower risk of recurrence and 66-71\% lower risk of mortality at six months of follow-up. After three years, a moderate level was still associated with a $45 \%$ lower risk of mortality, while there was no association between n-3 PUFA levels and recurrence risk. As the fatty acid composition in the erythrocyte membrane reflects the diet $(24,25)$, these findings suggest that a medium or high dietary intake of n-3 PUFAs may be associated with a better prognosis after VTE, although the effect on recurrence risk appeared to be transient (23). Apparently conflicting, we found that a high dietary intake of n3 PUFAs was associated with 55\% and 51\% lower recurrence risk after unprovoked VTE and DVT, respectively, and that the effect occurred almost two years after the incident event. Although the different exposure assessments hamper a direct comparison of the studies, there are some plausible explanations for the diverging findings. First, the n-3 PUFA intake our study was high, and the upper tertile cut-off equals more than four grams per day, which is higher than the doses used in most clinical trials on prevention (34). Moreover, Reiner et al. (23) included PUFAs derived from both marine sources (i.e. EPA, DPA and DHA) and plants (i.e. alpha-linolenic acid, ALA) in their exposure variable. The impact of ALA in relation to health outcomes is less investigated and an effect is probably modest compared to marinederived n-3 PUFAs (35-37). Therefore, the association between n-3 PUFAs and recurrence risk may have been underestimated due to the inclusion of ALA in the study by Reiner et al. (23). Further, the patients included in SWITCO65+ were almost ten years older than those in 
the present study (75 years vs. 67 years), and $29 \%$ of the patients had a history of previous VTE, while we exclusively included incident cases.

The beneficial association of n-3 PUFAs on recurrence risk in our study was restricted to those with a high weekly intake, suggesting a mild protective effect of n-3 PUFA intake. Stratified analyses further revealed that the association was mainly driven by an effect in patients with unprovoked incident events. The risk of recurrence largely depends on the etiology of the incident event, which also reflects the underlying predisposition (baseline risk) for thrombosis $(8,38)$. Assuming a mild protective effect of n-3 PUFAs on VTE recurrence, it is plausible that the effect is most prominent in those with an intermediate recurrence risk, such as patients with unprovoked VTE. Conversely, a small effect may be difficult to observe in those with a low risk (e.g. VTE provoked by major surgery), and may be overwhelmed in those with a high risk (e.g. VTE provoked by active or progressive cancer). This is supported by our sensitivity analyses showing lowered risk estimates, even for provoked events, when patients with active cancer were excluded.

We further observed a difference in the influence of n-3 PUFA intake on the risk of recurrence after DVT and PE. Apparently, a high intake was associated with a lower recurrence risk after DVT, and a higher risk of recurrence after PE. Although PE typically recur as a new PE and is regarded as a more severe form of VTE (39), the current guidelines for antithrombotic therapy do not distinguish between the two disease entities (9). However, in our data, which reflects clinical practice from 1994 to 2016, we found that $45 \%$ of patients with PE were scheduled anticoagulant treatment for $\geq 1$ year, while this applied to only $13 \%$ of patients with DVT. The risk of recurrence is highest during the first year after incident VTE $(4,5)$, and $53 \%$ of the recurrences in the present study occurred during this period. Potentially, DVT patients receiving anticoagulant treatment for a shorter time-period may have benefitted more from a high intake of n-3 PUFAs, while an effect in patients with PE 
may have been masked by long-term treatment. We addressed this point by restricting the follow-up time to the period after completion of anticoagulant treatment. Interestingly, this approach consistently showed inverse associations between high n-3 PUFA intake and recurrence after overall VTE and subtypes of VTE (unprovoked, provoked, DVT and PE).

In contrast to the findings by Reiner and colleagues (23), we did not observe any association between n-3 PUFAs and the risk of mortality in patients with VTE. Although observational studies on the effect of n-3 PUFAs on cause-specific mortality in individuals without known CVD have shown somewhat diverging results $(40,41)$, it may be suggested that marine-derived n-3 PUFAs are primarily associated with a lower risk of CVD-related death, while ALA is associated with a lower risk of death from non-CVD causes $(36,42)$. The most frequently reported causes of death in patients with VTE are cancer, PE, infections, and other cardiovascular or respiratory diseases $(3,43,44)$. Potentially, a relatively higher proportion of deaths due to non-CVD causes in VTE-patients could explain the lack of a beneficial association between marine n-3 PUFAs and all-cause mortality in our study. Nevertheless, the results did not change after exclusion of patients with active cancer.

The main strengths of our study include unselected VTE patients with a wide age distribution, a validated exposure variable, long follow-up and thoroughly validated outcomes. Limitations of the study include an observational design, which does not allow for random allocation to exposure groups, and residual confounding may be present. Further, a substantial proportion of participants were excluded due to incomplete information on n-3 PUFA intake, and the included participants were younger and healthier compared to those excluded. This may influence the generalizability of our findings. Moreover, although we previously reported a significant trend between self-reported intake of marine n-3 PUFAs and serum concentrations (22), the correlation coefficients are generally reported to be low (45). Due to limited study power, the confidence intervals were wide, and our findings should 
therefore be interpreted with caution. Finally, a long follow-up may introduce regression dilution as dietary habits may change over time. Such misclassification may influence the precision of our estimates, but is unlikely to be related the outcomes of the study, and would typically lead to an underestimation of the true association.

In conclusion, a high intake of marine n-3 PUFAs was associated with a lower risk of VTE recurrence, which was most pronounced after unprovoked VTE and DVT, and in cancerfree patients. In contrast, there was no association between n-3 PUFAs and the risk of allcause mortality in patients with incident VTE.

\section{CONFLICT OF INTEREST}

None declared.

\section{ACKNOWLEDGEMENTS}

The K.G. Jebsen - Thrombosis Research and Expertise Center is supported by an independent grant from Stiftelsen Kristian Gerhard Jebsen.

Figure 1 Ten-year cumulative incidence of recurrent venous thromboembolism (VTE) by tertiles of weekly intake of n-3 PUFAs for the total follow-up (Panel A) and follow-up restricted to the period after discontinuation of anticoagulant treatment (Panel B). Low intake: $<8.19 \mathrm{~g} / \mathrm{week}$, medium intake: 8.19-29.1 g/week, and high intake: >29.1 g/week. PUFAs polyunsaturated fatty acids. 
Figure 2 panel A-D. Cumulative incidence of recurrent venous thromboembolism (VTE) by tertiles of weekly intake of n-3 PUFAs according to the index event (A: unprovoked VTE, B: provoked VTE, C: Pulmonary embolism (PE), D: Deep vein thrombosis (DVT)). Low intake: <8.19 g/week, medium intake: 8.19-29.1 g/week and high intake: >29.1 g/week. 


\section{REFERENCES}

1. Cohen AT, Agnelli G, Anderson FA, et al. Venous thromboembolism (VTE) in Europe. The number of VTE events and associated morbidity and mortality. Thromb Haemost 2007; 98: 756-764.

2. Kearon C. Natural history of venous thromboembolism. Circulation 2003; 107: I22-130.

3. Naess IA, Christiansen SC, Romundstad P, et al. Incidence and mortality of venous thrombosis: a population-based study. J Thromb Haemost 2007; 5: 692-699.

4. Arshad N, Bjori E, Hindberg K, et al. Recurrence and mortality after first venous thromboembolism in a large population-based cohort. J Thromb Haemost 2016; 14: 1-9.

5. Heit JA, Mohr DN, Silverstein MD, et al. Predictors of recurrence after deep vein thrombosis and pulmonary embolism: a population-based cohort study. Arch Intern Med 2000; 160: 761-768.

6. Prandoni P, Lensing AW, Cogo A, et al. The long-term clinical course of acute deep venous thrombosis. Ann Intern Med 1996; 125: 1-7.

7. Sogaard KK, Schmidt M, Pedersen L, et al. 30-year mortality after venous thromboembolism: a population-based cohort study. Circulation 2014; 130: 829-836.

8. Kearon C, Ageno W, Cannegieter SC, et al. Categorization of patients as having provoked or unprovoked venous thromboembolism: guidance from the SSC of ISTH. J Thromb Haemost 2016; 14: 1480-1483.

9. Kearon C, Akl EA, Ornelas J, et al. Antithrombotic Therapy for VTE Disease: CHEST Guideline and Expert Panel Report. Chest 2016; 149: 315-352.

10. Heit JA, Lahr BD, Petterson TM, et al. Heparin and warfarin anticoagulation intensity as predictors of recurrence after deep vein thrombosis or pulmonary embolism: a population-based cohort study. Blood 2011; 118: 4992-4999. 
11. The Norwegian Directorate of Health. Kostråd om fisk og sjømat (Dietary advice on fish and seafood). Available at: https://helsenorge.no/kosthold-og-ernaring/kostrad/spis-

fisk-oftere. Accessed June 22, 2019.

12. U.S. Department of Health and Human Services and U.S. Department of Agriculture. 2015 - 2020 Dietary Guidelines for Americans. 8th edition,. Available at: https://health.gov/dietaryguidelines/2015/guidelines/ Accessed June 22, 2018.

13. Rimm EB, Appel LJ, Chiuve SE, et al. Seafood Long-Chain n-3 Polyunsaturated Fatty Acids and Cardiovascular Disease: A Science Advisory From the American Heart Association. Circulation 2018; 138: 35-47.

14. Surette ME. The science behind dietary omega-3 fatty acids. CMAJ 2008; 178: 177180.

15. Hansen JB, Olsen JO, Wilsgård L, et al. Comparative effects of prolonged intake of highly purified fish oils as ethyl ester or triglyceride on lipids, haemostasis and platelet function in normolipaemic men. Eur J Clin Nutr 1993; 47: 497-507.

16. Hansen JB, Svensson B, Wilsgard L, et al. Serum enriched with n-3 polyunsaturated fatty acids inhibits procoagulant activity in endothelial cells. Blood Coagul Fibrinolysis 1991; 2: 515-519.

17. De Caterina R, Cybulsky MA, Clinton SK, et al. Omega-3 fatty acids and endothelial leukocyte adhesion molecules. Prostaglandins Leukot Essent Fatty Acids 1995; 52: 191195.

18. Moertl D, Berger R, Hammer A, et al. Dose-dependent decrease of platelet activation and tissue factor by omega-3 polyunsaturated fatty acids in patients with advanced chronic heart failure. Thromb Haemost 2011; 106: 457-465. 
19. Hansen-Krone IJ, Enga KF, Sudduth-Klinger JM, et al. High fish plus fish oil intake is associated with slightly reduced risk of venous thromboembolism: the Tromso Study. J Nutr 2014; 144: 861-867.

20. Severinsen MT, Overvad K, Andersen VL, et al. Fish intake and venous thromboembolism: a Danish follow-up study. Thromb Res 2014; 133: 352-356.

21. Steffen LM, Folsom AR, Cushman M, et al. Greater fish, fruit, and vegetable intakes are related to lower incidence of venous thromboembolism: the Longitudinal Investigation of Thromboembolism Etiology. Circulation 2007; 115: 188-195.

22. Isaksen T, Evensen LH, Johnsen SH, et al. Dietary intake of marine n-3 polyunsaturated fatty acids and future risk of venous thromboembolism. Res Pract Thromb Haemost 2019; 3: 59-69.

23. Reiner MF, Stivala S, Limacher A, et al. Omega-3 Fatty Acids Predict Recurrent Venous Thromboembolism or Total Mortality in Elderly Patients with Acute Venous Thromboembolism. J Thromb Haemost 2016; 15: 47-57.

24. Sarkkinen ES, Agren JJ, Ahola I, et al. Fatty acid composition of serum cholesterol esters, and erythrocyte and platelet membranes as indicators of long-term adherence to fat-modified diets. Am J Clin Nutr 1994; 59: 364-370.

25. Simopoulos AP. Omega-3 fatty acids in health and disease and in growth and development. Am J Clin Nutr 1991; 54: 438-463.

26. Jacobsen BK, Eggen AE, Mathiesen EB, et al. Cohort profile: the Tromso Study. Int J Epidemiol 2012; 41: 961-967.

27. Braekkan SK, Mathiesen EB, Njolstad I, et al. Family history of myocardial infarction is an independent risk factor for venous thromboembolism: the Tromso study. J Thromb Haemost 2008; 6: 1851-1857. 
28. Jacobsen BK, Nilsen H. High education is associated with low fat and high fibre, betacarotene and vitamin C. Computation of nutrient intake based on a short food frequency questionnaire in 17,256 men and women in the Troms $\emptyset$ Study. Nor Epidemiol 2000; 10: $57-62$.

29. Institute of Marine Research. Seafood data Available at: https://www.nifes.no/. Accessed August 17, 2018.

30. Norwegian Food Safety Authority. Matvaretabellen 2018 (Table of food composition 2018). Available at: http://www.matvaretabellen.no/ Accessed August 17, 2018.

31. Fine JP, Gray RJ. A proportional hazards model for the subdistribution of a competing risk. J Am Stat Assoc 1999; 94: 496-509.

32. Hansen JB, Olsen JO, Wilsgard L, et al. Effects of dietary supplementation with cod liver oil on monocyte thromboplastin synthesis, coagulation and fibrinolysis. J Intern Med Suppl 1989; 731: 133-139.

33. Vanschoonbeek K, Wouters K, van der Meijden PE, et al. Anticoagulant effect of dietary fish oil in hyperlipidemia: a study of hepatic gene expression in APOE2 knockin mice. Arterioscler Thromb Vasc Biol 2008; 28: 2023-2029.

34. Aung T, Halsey J, Kromhout D, et al. Associations of omega-3 fatty acid supplement use with cardiovascular disease risks: Meta-analysis of 10 trials involving 77917 individuals. JAMA Cardiology 2018.

35. Mozaffarian D, Wu JH. Omega-3 fatty acids and cardiovascular disease: effects on risk factors, molecular pathways, and clinical events. J Am Coll Cardiol 2011; 58: 20472067.

36. Sala-Vila A, Guasch-Ferre M, Hu FB, et al. Dietary alpha-Linolenic Acid, Marine omega-3 Fatty Acids, and Mortality in a Population With High Fish Consumption: 
Findings From the PREvencion con DIeta MEDiterranea (PREDIMED) Study. J Am Heart Assoc 2016; 5.

37. Pan A, Chen M, Chowdhury R, et al. alpha-Linolenic acid and risk of cardiovascular disease: a systematic review and meta-analysis. Am J Clin Nutr 2012; 96: 1262-1273.

38. Rosendaal FR. Venous thrombosis: a multicausal disease. Lancet 1999; 353: 11671173.

39. Arshad N, Bjori E, Hindberg K, et al. Recurrence and mortality after first venous thromboembolism in a large population-based cohort. J Thromb Haemost 2017; 15: 295-303.

40. Alexander DD, Miller PE, Van Elswyk ME, et al. A Meta-Analysis of Randomized Controlled Trials and Prospective Cohort Studies of Eicosapentaenoic and Docosahexaenoic Long-Chain Omega-3 Fatty Acids and Coronary Heart Disease Risk. Mayo Clin Proc 2017; 92: 15-29.

41. Aung T, Halsey J, Kromhout D, et al. Associations of Omega-3 Fatty Acid Supplement Use With Cardiovascular Disease Risks: Meta-analysis of 10 Trials Involving 77917 Individuals. JAMA Cardiol 2018; 3: 225-234.

42. Mozaffarian D, Lemaitre RN, King IB, et al. Plasma phospholipid long-chain omega-3 fatty acids and total and cause-specific mortality in older adults: a cohort study. Ann Intern Med 2013; 158: 515-525.

43. Flinterman LE, van Hylckama Vlieg A, Cannegieter SC, et al. Long-term survival in a large cohort of patients with venous thrombosis: incidence and predictors. PLoS Med 2012; 9: e1001155.

44. Faller N, Limacher A, Mean M, et al. Predictors and Causes of Long-Term Mortality in Elderly Patients with Acute Venous Thromboembolism: A Prospective Cohort Study. Am J Med 2017; 130: 198-206. 
45. von Schacky C. Omega-3 fatty acids in cardiovascular disease--an uphill battle.

Prostaglandins Leukot Essent Fatty Acids 2015; 92: 41-47. 


\section{TABLES AND FIGURES}

Table 1 Characteristics of patients with incident VTE ( $n=595)$ and recurrent events $(n=98)$ according to tertiles of weekly intake of n-3 PUFAs. The Tromsø Study (1994-2016)

\begin{tabular}{|c|c|c|c|c|}
\hline n-3 PUFAs (g/week) & All & T1 $(<8.19)$ & T2 (8.19-29.1) & T3 $(>29.1)$ \\
\hline \multicolumn{5}{|l|}{ First VTE } \\
\hline Number of patients & 595 & 200 & 201 & 194 \\
\hline DVT & $57.5(342)$ & $53.0(106)$ & $63.2(127)$ & $56.2(109)$ \\
\hline PE & $42.5(253)$ & $47.0(94)$ & $36.8(74)$ & $43.8(85)$ \\
\hline Unprovoked & $38.0(226)$ & $42.0(84)$ & $47.8(96)$ & $42.3(82)$ \\
\hline \multicolumn{5}{|c|}{ Duration of anticoagulant treatment } \\
\hline$<3$ months & 20.0 (119) & $31.9(38)$ & $37.0(44)$ & $31.1(37)$ \\
\hline 3-6 months & $42.5(253)$ & $30.4(77)$ & $35.2(89)$ & $34.4(87)$ \\
\hline 6-12 months & $20.5(122)$ & $45.1(55)$ & $29.5(36)$ & $25.4(31)$ \\
\hline$>12$ months & $17.0(101)$ & $29.7(30)$ & $31.7(32)$ & $38.6(39)$ \\
\hline Male sex & $51.1(304)$ & $54.5(109)$ & $47.8(96)$ & $51.0(99)$ \\
\hline Age at incident VTE & $67 \pm 13$ & $63 \pm 14$ & $67 \pm 13$ & $71 \pm 12$ \\
\hline BMI, $\mathrm{kg} / \mathrm{m}^{2}$ & $27.4 \pm 4.5$ & $27.1 \pm 4.3$ & $27.4 \pm 4.3$ & $27.7 \pm 4.4$ \\
\hline Diabetes & $4.9(29)$ & $3.0(6)$ & $6.0(12)$ & $5.7(11)$ \\
\hline History of CVD & $13.1(78)$ & $7.5(15)$ & $14.4(29)$ & $17.5(34)$ \\
\hline Cancer & $16.8(100)$ & $18.0(36)$ & $13.9(28)$ & $18.6(36)$ \\
\hline Current smoking & $28.2(168)$ & $40.0(80)$ & $26.9(54)$ & $17.5(34)$ \\
\hline Systolic BP, mmHg & $142 \pm 24$ & $138 \pm 23$ & $142 \pm 24$ & $147 \pm 25$ \\
\hline \multicolumn{5}{|c|}{ Second VTE (by index event): } \\
\hline Number of patients & 98 & 35 & 38 & 25 \\
\hline DVT & $72.4(71)$ & $77.1(27)$ & $81.6(31)$ & $52.0(13)$ \\
\hline PE & $27.6(27)$ & $22.9(8)$ & $18.4(7)$ & $48.0(12)$ \\
\hline Unprovoked & $52.0(51)$ & $51.4(18)$ & $63.2(24)$ & $36.0(9)$ \\
\hline Male sex & $61.2(60)$ & $77.1(27)$ & $55.3(21)$ & $48.0(12)$ \\
\hline Age at second VTE & $69 \pm 12$ & $67 \pm 13$ & $69 \pm 12$ & $71 \pm 12$ \\
\hline BMI, $\mathrm{kg} / \mathrm{m}^{2}$ & $27.0 \pm 4.5$ & $27.1 \pm 6.0$ & $27.0 \pm 3.3$ & $26.8 \pm 3.9$ \\
\hline Cancer & $18.4(18)$ & $11.4(4)$ & $13.2(5)$ & $36.0(9)$ \\
\hline Current smoking & $20.4(20)$ & $31.4(11)$ & $23.7(9)$ & $20.0(5)$ \\
\hline Systolic BP, mmHg & $139 \pm 20$ & $135 \pm 20$ & $143 \pm 20$ & $139 \pm 21$ \\
\hline
\end{tabular}


$B M I$ body mass index, $B P$ blood pressure, $C V D$ cardiovascular disease (angina pectoris, stroke or myocardial infarction), $D V T$ deep vein thrombosis, $P E$ pulmonary embolism, $P U F A s$ polyunsaturated fatty acids, $T$ tertile, VTE venous thromboembolism.

Values are mean (standard deviation) or percentage (count) 
Table 2 Incidence rates (IRs) and hazard ratios (HRs) with 95\% confidence intervals (CI) for recurrent venous thromboembolism (VTE) by tertiles of weekly intake of marine n-3 polyunsaturated fatty acids (PUFAs). The Tromsø Study 1994-2016.

\begin{tabular}{|c|c|c|c|c|c|c|}
\hline $\begin{array}{l}\text { n-3 PUFA intake } \\
\text { (g/week) }\end{array}$ & $\begin{array}{l}\text { Person- } \\
\text { years }\end{array}$ & $\begin{array}{l}\text { VTE- } \\
\text { events }\end{array}$ & $\begin{array}{c}\text { Crude IR } \\
(95 \% \text { CI })^{*}\end{array}$ & $\begin{array}{c}\text { Model 1 } \\
\text { HR }(95 \% \text { CI }) \dagger\end{array}$ & $\begin{array}{c}\text { Model } 2 \\
\text { HR }(95 \% \text { CI }) \neq\end{array}$ & $\begin{array}{c}\text { SHR } \\
(95 \% \mathrm{CI}) \S\end{array}$ \\
\hline \multicolumn{7}{|l|}{ VTE } \\
\hline $\mathrm{T} 1<8.19$ & 1111 & 35 & $3.1(2.3-4.4)$ & Ref. & Ref. & Ref. \\
\hline T2 $8.19-29.1$ & 1080 & 38 & $3.5(2.6-4.8)$ & $1.10(0.69-1.74)$ & $1.09(0.69-1.74)$ & $1.19(0.76-1.87)$ \\
\hline $\mathrm{T} 3>29.1$ & 916 & 25 & $2.7(3.7-4.0)$ & $0.86(0.51-1.43)$ & $0.77(0.45-1.30)$ & $0.83(0.49-1.42)$ \\
\hline \multicolumn{7}{|l|}{ Unprovoked VTE } \\
\hline $\mathrm{T} 1<8.19$ & 537 & 18 & $3.4(2.1-5.3)$ & Ref. & Ref. & Ref. \\
\hline T2 $8.19-29.1$ & 591 & 24 & $4.1(2.7-6.1)$ & $1.20(0.65-2.21)$ & $1.13(0.61-2.10)$ & $1.12(0.61-2.08)$ \\
\hline $\mathrm{T} 3>29.1$ & 448 & 9 & $2.0(1.0-3.9)$ & $0.59(0.26-1.31)$ & $0.45(0.20-1.01)$ & $0.42(0.18-1.00)$ \\
\hline \multicolumn{7}{|l|}{ Provoked VTE } \\
\hline $\mathrm{T} 1<8.19$ & 575 & 17 & $3.0(1.8-4.8)$ & Ref. & Ref. & Ref. \\
\hline T2 $8.19-29.1$ & 487 & 14 & $2.9(1.7-4.9)$ & $0.96(0.47-1.96)$ & $1.00(0.49-2.04)$ & $1.10(0.56-2.19)$ \\
\hline $\mathrm{T} 3>29.1$ & 468 & 16 & $3.4(2.1-5.6)$ & $1.21(0.60-2.43)$ & $1.25(0.61-2.56)$ & $1.35(0.70-2.61)$ \\
\hline \multicolumn{7}{|l|}{ PE } \\
\hline $\mathrm{T} 1<8.19$ & 540 & 8 & $1.5(0.7-3.0)$ & Ref. & Ref. & Ref. \\
\hline T2 $8.19-29.1$ & 378 & 7 & $1.9(0.9-3.9)$ & $1.18(0.43-3.27)$ & $0.97(0.35-2.71)$ & $1.10(0.41-2.95)$ \\
\hline $\mathrm{T} 3>29.1$ & 405 & 12 & $3.0(1.7-5.2)$ & $1.93(0.78-4.75)$ & $1.55(0.62-3.87)$ & $1.64(0.65-4.12)$ \\
\hline \multicolumn{7}{|l|}{ DVT } \\
\hline $\mathrm{T} 1<8.19$ & 571 & 27 & $4.7(3.2-6.9)$ & Ref. & Ref. & Ref. \\
\hline T2 $8.19-29.1$ & 703 & 31 & $4.4(3.1-6.3)$ & $0.94(0.56-1.57)$ & $1.00(0.59-1.69)$ & $1.12(0.67-1.86)$ \\
\hline $\mathrm{T} 3>29.1$ & 511 & 13 & $2.5(1.5-4.4)$ & $0.54(0.28-1.06)$ & $0.49(0.24-0.97)$ & $0.54(0.27-1.08)$ \\
\hline
\end{tabular}

CI confidence interval, $D V T$ deep vein thrombosis, $P E$ pulmonary embolism, SHR subdistribution hazard ratio, $T$ tertile.

*Per 100 person-years

†Unadjusted

\$Adjusted for age, sex and body mass index

$\S$ Competing risk model, adjusted for age, sex and body mass index 
Table 3 Incidence rates (IRs) and hazard ratios (HRs) for recurrent venous thromboembolism (VTE) by tertiles of weekly intake of marine n-3 polyunsaturated fatty acids (n-3 PUFAs) after excluding individuals with active cancer. The Tromsø Study 1994-2016

\begin{tabular}{|c|c|c|c|c|c|c|}
\hline $\begin{array}{l}\text { n-3 PUFA intake } \\
\text { (g/week) }\end{array}$ & $\begin{array}{c}\text { Person- } \\
\text { years }\end{array}$ & $\begin{array}{c}\text { VTE- } \\
\text { events }\end{array}$ & $\begin{array}{c}\text { Crude IR } \\
(\mathbf{9 5 \%} \text { CI })^{*}\end{array}$ & $\begin{array}{c}\text { Model } 1 \\
\text { HR }(95 \% \text { CI }) \dagger \\
\end{array}$ & $\begin{array}{c}\text { Model 2 } \\
\text { HR }(95 \% \text { CI) }\end{array}$ & $\begin{array}{c}\text { SHR } \\
(95 \% \text { CI }) \S \\
\end{array}$ \\
\hline \multicolumn{7}{|l|}{ VTE } \\
\hline $\mathrm{T} 1<8.19$ & 1026 & 31 & $3.0(2.1-4.3)$ & Ref. & Ref. & Ref. \\
\hline T2 $8.19-29.1$ & 1005 & 33 & $3.3(2.3-4.6)$ & $1.07(0.65-1.74)$ & $1.01(0.61-1.66)$ & $1.09(0.67-1.77)$ \\
\hline $\mathrm{T} 3>29.1$ & 826 & 16 & $1.9(1.2-3.2)$ & $0.64(0.35-1.17)$ & $0.51(0.27-0.95)$ & $0.56(0.30-1.03)$ \\
\hline \multicolumn{7}{|l|}{ Unprovoked VTE } \\
\hline $\mathrm{T} 1<8.19$ & 524 & 16 & $3.1(1.9-5.0)$ & Ref. & Ref. & Ref. \\
\hline T2 $8.19-29.1$ & 570 & 21 & $3.7(2.4-5.7)$ & $1.20(0.62-2.29)$ & $1.13(0.59-2.18)$ & $1.15(0.60-2.22)$ \\
\hline $\mathrm{T} 3>29.1$ & 407 & 7 & $1.7(0.8-3.6)$ & $0.55(0.23-1.34)$ & $0.41(0.17-1.03)$ & $0.38(0.15-1.00)$ \\
\hline \multicolumn{7}{|l|}{ Provoked VTE } \\
\hline $\mathrm{T} 1<8.19$ & 502 & 15 & $3.0(1.8-5.0)$ & Ref. & Ref. & Ref. \\
\hline T2 8.19-29.1 & 433 & 12 & $2.8(1.6-4.9)$ & $0.92(0.43-1.96)$ & $0.88(0.40-1.90)$ & $0.97(0.47-2.04)$ \\
\hline $\mathrm{T} 3>29.1$ & 419 & 9 & $2.1(1.1-4.1)$ & $0.76(0.33-1.77)$ & $0.66(0.27-1.57)$ & $0.79(0.36-1.73)$ \\
\hline \multicolumn{7}{|l|}{ PE } \\
\hline $\mathrm{T} 1<8.19$ & 500 & 8 & $1.6(0.8-3.2)$ & Ref. & Ref. & Ref. \\
\hline T2 8.19-29.1 & 346 & 6 & $1.7(0.8-3.9)$ & $1.02(0.35-2.96)$ & $0.75(0.26-2.18)$ & $0.91(0.33-2.52)$ \\
\hline $\mathrm{T} 3>29.1$ & 354 & 8 & $2.3(1.3-4.5)$ & $1.35(0.50-3.62)$ & $0.99(0.36-2.71)$ & $1.07(0.41-2.81)$ \\
\hline \multicolumn{7}{|l|}{ DVT } \\
\hline $\mathrm{T} 1<8.19$ & 526 & 23 & $4.4(2.9-6-6)$ & Ref. & Ref. & Ref. \\
\hline T2 8.19-29.1 & 660 & 27 & $4.1(2.8-6.0)$ & $0.93(0.53-1.63)$ & $0.98(0.55-1.73)$ & $1.04(0.60-1.81)$ \\
\hline $\mathrm{T} 3>29.1$ & 472 & 8 & $1.7(0.8-3.4)$ & $0.39(0.17-0.88)$ & $0.31(0.13-0.71)$ & $0.35(0.15-0.81)$ \\
\hline
\end{tabular}

$C I$ confidence interval, $D V T$, deep vein thrombosis, $P E$ pulmonary embolism, $S H R$ subdistribution hazard ratio, $T$ tertile.

*Per 100 person-years

$\dagger$ †nadjusted

$\$$ Adjusted for age, sex and body mass index

$\S$ Competing risk model, adjusted for age, sex and body mass index 
Table 4 Mortality rates (MRs) and hazard ratios (HRs) for all-cause mortality across tertiles of weekly intake of marine n-3 polyunsaturated fatty acids (n-3 PUFAs). The Troms $\varnothing$ Study 1994-2016.

\begin{tabular}{|c|c|c|c|c|c|}
\hline $\begin{array}{l}\text { n-3 PUFA intake } \\
\text { (g/week) }\end{array}$ & $\begin{array}{l}\text { Person- } \\
\text { years }\end{array}$ & Deaths & $\begin{array}{c}\text { Crude MR (95\% } \\
\text { CI) })^{*}\end{array}$ & $\begin{array}{c}\text { Model 1 } \\
\text { HR }(95 \% \text { CI }) \dagger \\
\end{array}$ & $\begin{array}{c}\text { Model } 2 \\
\text { HR }(95 \% \text { CI }) \ddagger\end{array}$ \\
\hline \multicolumn{6}{|l|}{ VTE } \\
\hline $\mathrm{T} 1<8.19$ & 1302 & 72 & $5.5(4.4-7.0)$ & Ref. & Ref. \\
\hline T2 8.19-29.1 & 1220 & 76 & $6.2(5.0-7.8)$ & $0.97(0.70-1.34)$ & $0.96(0.69-1.35)$ \\
\hline T3 > 29.1 & 1033 & 79 & $7.6(6.1-9.5)$ & $1.01(0.72-1.40)$ & $1.02(0.73-1.43)$ \\
\hline \multicolumn{6}{|l|}{ Unprovoked VTE } \\
\hline $\mathrm{T} 1<8.19$ & 658 & 17 & $2.6(1.6-4.2)$ & Ref. & Ref. \\
\hline T2 $8.19-29.1$ & 682 & 24 & $3.5(2.4-5.3)$ & $1.11(0.59-2.08)$ & $1.08(0.56-2.10)$ \\
\hline T3 $>29.1$ & 510 & 26 & $5.1(3.5-7.5)$ & $1.26(0.68-2.35)$ & $1.26(0.67-2.36)$ \\
\hline \multicolumn{6}{|l|}{ Provoked VTE } \\
\hline $\mathrm{T} 1<8.19$ & 644 & 55 & $8.5(6.6-11.1)$ & Ref. & Ref. \\
\hline T2 8.19-29.1 & 537 & 51 & $9.5(7.2-12.5)$ & $0.98(0.66-1.44)$ & $1.02(0.69-1.52)$ \\
\hline $\mathrm{T} 3>29.1$ & 523 & 53 & $10.1(7.7-13.3)$ & $0.91(0.61-1.35)$ & $0.94(0.62-1.42)$ \\
\hline \multicolumn{6}{|l|}{ PE } \\
\hline $\mathrm{T} 1<8.19$ & 586 & 29 & $4.9(3.4-7.1)$ & Ref. & Ref. \\
\hline T2 $8.19-29.1$ & 403 & 23 & $5.7(3.8-8.6)$ & $0.95(0.54-1.68)$ & $0.95(0.53-1.68)$ \\
\hline $\mathrm{T} 3>29.1$ & 457 & 35 & $7.7(5.5-10.7)$ & $1.21(0.73-2.02)$ & $1.16(0.69-1.96)$ \\
\hline \multicolumn{6}{|l|}{ DVT } \\
\hline $\mathrm{T} 1<8.19$ & 716 & 43 & $6.0(4.5-8.1)$ & Ref. & Ref. \\
\hline T2 $8.19-29.1$ & 818 & 53 & $6.5(5.0-8.5)$ & $0.95(0.63-1.44)$ & $0.98(0.64-1.49)$ \\
\hline $\mathrm{T} 3>29.1$ & 575 & 44 & $7.6(5.7-10.3)$ & $0.87(0.56-1.35)$ & $0.94(0.60-1.48)$ \\
\hline
\end{tabular}

$C I$ confidence interval, $D V T$ deep vein thrombosis, $P E$ pulmonary embolism, $T$ tertile.

*Per 100 person-years

$\dagger$ Adjusted for age (as time-scale)

$¥$ Model $1+$ sex, body mass index, systolic blood pressure and smoking 
Table 5 Mortality rates (MRs) and hazard ratios (HRs) for all-cause mortality by tertiles of weekly intake of marine n-3 polyunsaturated fatty acids (n-3 PUFAs) excluding individuals with active cancer. The Tromsø Study 1994-2016.

\begin{tabular}{|c|c|c|c|c|c|}
\hline $\begin{array}{l}\text { n-3 PUFA intake } \\
\text { (g/week) }\end{array}$ & $\begin{array}{c}\text { Person- } \\
\text { years }\end{array}$ & Deaths & $\begin{array}{l}\text { Crude MR } \\
(95 \% \text { CI })^{*}\end{array}$ & $\begin{array}{c}\text { Model 1 } \\
\text { HR }(95 \% \text { CI }) \dagger\end{array}$ & $\begin{array}{c}\text { Model } 2 \\
\text { HR }(95 \% \text { CI }) \ddagger\end{array}$ \\
\hline \multicolumn{6}{|l|}{ VTE } \\
\hline $\mathrm{T} 1<8.19$ & 1203 & 43 & $3.6(2.7-4.8)$ & Ref. & Ref. \\
\hline T2 $8.19-29.1$ & 1138 & 53 & $4.7(3.6-6.1)$ & $1.03(0.68-1.55)$ & $1.00(0.66-1.51)$ \\
\hline $\mathrm{T} 3>29.1$ & 921 & 51 & $5.5(4.2-7.3)$ & $0.96(0.63-1.45)$ & $0.91(0.60-1.40)$ \\
\hline \multicolumn{6}{|l|}{ Unprovoked VTE } \\
\hline $\mathrm{T} 1<8.19$ & 631 & 14 & $2.2(1.3-3.7)$ & Ref. & Ref. \\
\hline T2 $8.19-29.1$ & 653 & 17 & $2.6(1.6-4.2)$ & $0.95(0.46-1.95)$ & $0.92(0.43-1.97)$ \\
\hline $\mathrm{T} 3>29.1$ & 456 & 22 & $4.8(3.2-7.3)$ & $1.34(0.68-2.64)$ & $1.28(0.64-2.57)$ \\
\hline \multicolumn{6}{|l|}{ Provoked VTE } \\
\hline $\mathrm{T} 1<8.19$ & 572 & 29 & $5.1(3.5-7.3)$ & Ref. & Ref. \\
\hline T2 $8.19-29.1$ & 483 & 35 & $7.3(5.2-10.1)$ & $1.09(0.66-1.80)$ & $1.04(0.62-1.74)$ \\
\hline $\mathrm{T} 3>29.1$ & 465 & 29 & $6.2(4.3-9.0)$ & $0.75(0.43-1.29)$ & $0.68(0.39-1.20)$ \\
\hline \multicolumn{6}{|l|}{ PE } \\
\hline $\mathrm{T} 1<8.19$ & 546 & 18 & $3.3(2.1-5.2)$ & Ref. & Ref. \\
\hline T2 $8.19-29.1$ & 368 & 14 & $3.8(2.3-6.4)$ & $0.88(0.42-1.83)$ & $0.86(0.41-1.80)$ \\
\hline $\mathrm{T} 3>29.1$ & 401 & 23 & $5.7(3.8-8.6)$ & $1.33(0.71-2.51)$ & $1.21(0.63-2.30)$ \\
\hline \multicolumn{6}{|l|}{ DVT } \\
\hline $\mathrm{T} 1<8.19$ & 657 & 25 & $3.8(2.6-5.6)$ & Ref. & Ref. \\
\hline T2 $8.19-29.1$ & 770 & 39 & $5.1(3.7-6.9)$ & $1.05(0.63-1.76)$ & $1.11(0.66-1.88)$ \\
\hline $\mathrm{T} 3>29.1$ & 520 & 28 & $5.4(3.7-7.8)$ & $0.75(0.43-1.31)$ & $0.80(0.45-1.41)$ \\
\hline
\end{tabular}

$C I$ confidence interval, $D V T$ deep vein thrombosis, $P E$ pulmonary embolism, $T$ tertile.

*Per 100 person-years

$\dagger$ Adjusted for age (as time-scale)

\$Model $1+$ sex, body mass index, systolic blood pressure and smoking 
Figure 1 Ten-year cumulative incidence of recurrent venous thromboembolism (VTE) by tertiles of weekly intake of n-3 PUFAs for the total follow-up (Panel A) and follow-up restricted to the period after discontinuation of anticoagulant treatment (Panel B). Low intake: $<8.19 \mathrm{~g} /$ week, medium intake: 8.19-29.1 g/week, and high intake: $>29.1 \mathrm{~g} /$ week. PUFAs polyunsaturated fatty acids.
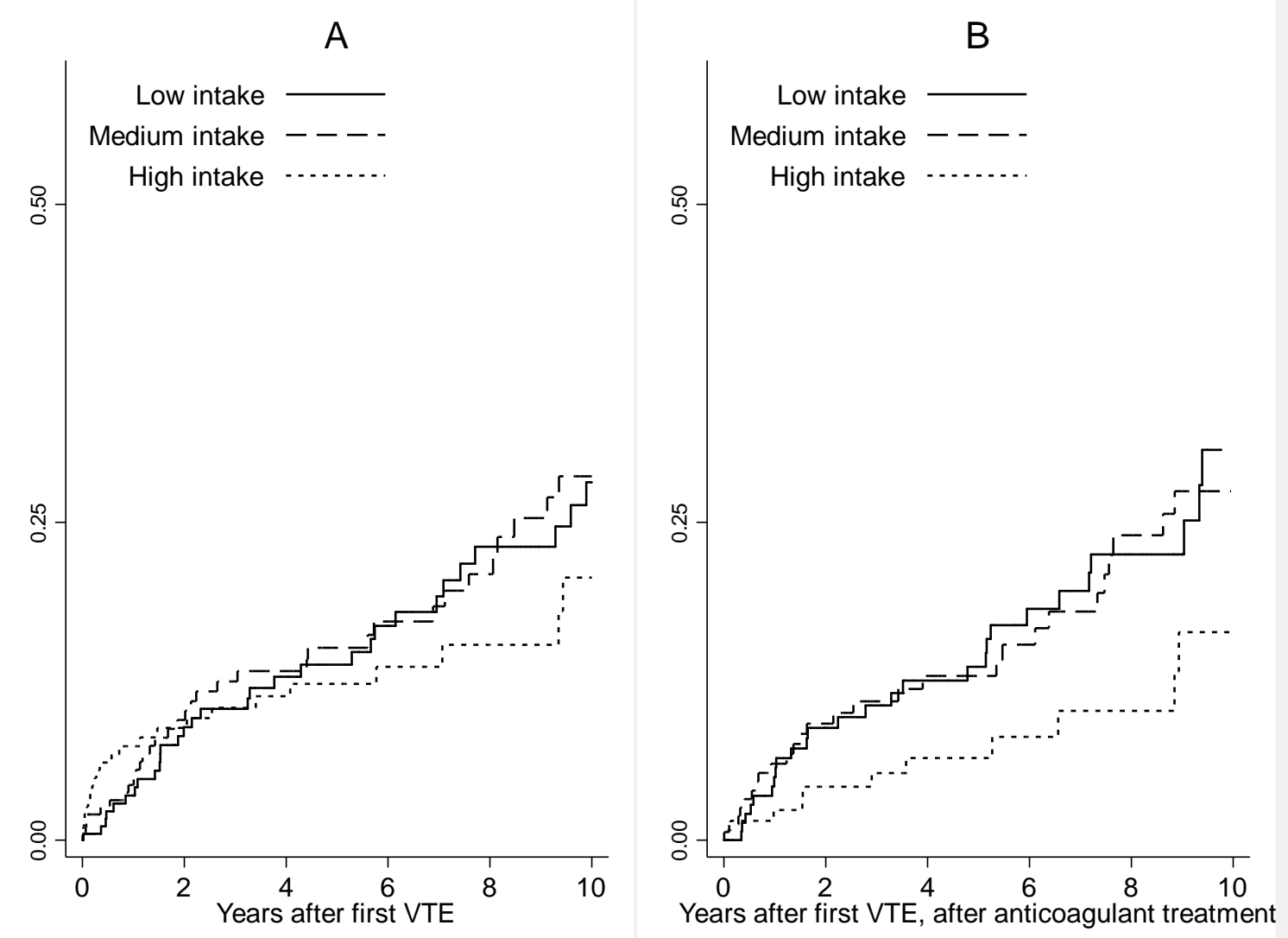
Figure 2 panel A-D. Cumulative incidence of recurrent venous thromboembolism (VTE) by tertiles of weekly intake of n-3 PUFAs according to the index event (A: unprovoked VTE, B: provoked VTE, C: Pulmonary embolism (PE), D: Deep vein thrombosis (DVT)). Low intake: $<8.19 \mathrm{~g} /$ week, medium intake: 8.19-29.1 g/week and high intake: >29.1 g/week.
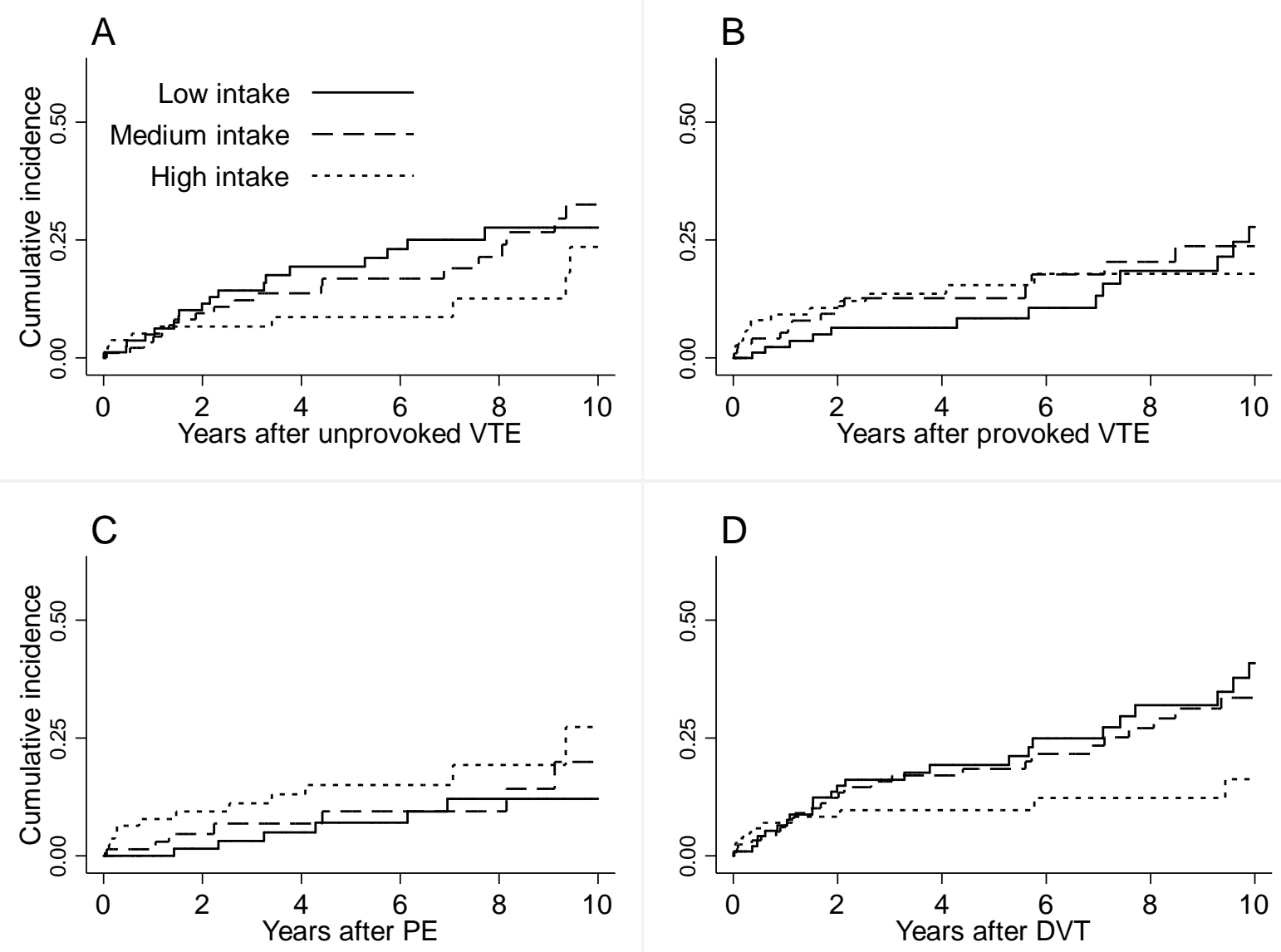
SUPPLEMENTARY MATERIAL

Table S1 Comparison of included and excluded VTE-patients

\begin{tabular}{lcc}
\hline & Included (n=595) & Excluded (n=304 \\
\hline Incident VTE & $57(342)$ & \\
DVT & $43(253)$ & $62(187)$ \\
PE & $44(262)$ & $38(117)$ \\
$\quad$ Unprovoked & $51(304)$ & $41(124)$ \\
Male sex & $67 \pm 13$ & $42(129)$ \\
Age at incident VTE & $27.4 \pm 4.5$ & $71 \pm 13$ \\
Body mass index, $\mathrm{kg} / \mathrm{m}^{2}$ & $5(29)$ & $27.1 \pm 4.5$ \\
Diabetes & $13(78)$ & $3(10)$ \\
History of CVD & $17(100)$ & $18(55)$ \\
Cancer & & $16(48)$
\end{tabular}

$\overline{C V D}$ cardiovascular disease (angina pectoris, stroke or myocardial infarction), $D V T$ deep vein thrombosis, $P E$ pulmonary embolism, VTE venous thromboembolism.

Values are mean (standard deviation) or percentage (count) 


\section{Table S2}

Incidence rates (IRs) and hazard ratios (HRs) with 95\% confidence intervals (CI) for recurrent venous thromboembolism (VTE) by tertiles of weekly intake of marine n-3 polyunsaturated fatty acids (PUFAs) in sensitivity analyses with follow-up restricted to the period after discontinuation of anticoagulant therapy. The Tromsø Study 1994-2016.

\begin{tabular}{lllllll}
\hline $\begin{array}{l}\text { n-3 PUFA intake } \\
\text { (g/week) }\end{array}$ & $\begin{array}{c}\text { Person- } \\
\text { years }\end{array}$ & $\begin{array}{c}\text { VTE- } \\
\text { events }\end{array}$ & $\begin{array}{c}\text { Crude IR } \\
(\mathbf{9 5 \%} \text { CI)* }\end{array}$ & $\begin{array}{c}\text { Model 1 } \\
\text { HR (95\% CI) } \dagger\end{array}$ & $\begin{array}{c}\text { Model 2 } \\
\text { HR (95\% CI)* }\end{array}$ & $\begin{array}{c}\text { SHR } \\
(\mathbf{9 5 \%} \text { CI)§ }\end{array}$ \\
\hline VTE & & & & & & \\
T 1 & 935 & 31 & $3.3(2.3-4.7)$ & Ref. & Ref. & Ref. \\
T 2 & 927 & 32 & $3.5(2.4-4.9)$ & $1.03(0.63-1.69)$ & $0.99(0.60-1.63)$ & $1.03(0.62-1.68)$ \\
T 3 & 747 & 13 & $1.7(1.0-3.0)$ & $0.53(0.28-1.02)$ & $0.42(0.21-0.82)$ & $0.48(0.25-0.94)$
\end{tabular}

\section{Unprovoked VTE}

$\begin{array}{lllllll}\text { T 1 } & 425 & 15 & 3.5(2.1-5.9) & \text { Ref. } & \text { Ref. } & \text { Ref. } \\ \text { T 2 } & 502 & 22 & 4.4(2.9-6.7) & 1.24(0.64-2.40) & 1.14(0.58-2.23) & 1.18(0.61-2.30) \\ \text { T 3 } & 361 & 6 & 1.7(0.7-3.7) & 0.48(0.19-1.25) & 0.31(0.12-0.83) & 0.33(0.12-0.89)\end{array}$

\section{Provoked VTE}

$\begin{array}{lllllll}\text { T 1 } & 511 & 16 & 3.1(1.9-5.1) & \text { Ref. } & \text { Ref. } & \text { Ref. } \\ \text { T 2 } & 424 & 10 & 2.4(1.3-4.4) & 0.76(0.34-1.68) & 0.79(0.35-1.77) & 0.77(0.35-1.69) \\ \text { T 3 } & 386 & 7 & 1.8(0.9-3.8) & 0.63(0.26-1.56) & 0.61(0.24-1.55) & 0.69(0.29-1.64) \\ \text { PE } & & & & & & \\ \text { T 1 } & 415 & 8 & 1.9(1.0-3.9) & \text { Ref. } & \text { Ref. } & \text { Ref. } \\ \text { T 2 } & 294 & 6 & 2.0(0.9-4.5) & 1.02(0.35-2.95) & 0.67(0.23-1.95) & 0.74(0.26-2.15) \\ \text { T 3 } & 321 & 6 & 1.9(0.8-4.2) & 0.99(0.34-2.87) & 0.70(0.24-2.04) & 0.82(0.29-2.32)\end{array}$

\section{DVT}

$\begin{array}{lllllll}\text { T 1 } & 521 & 23 & 4.4(2.9-6.6) & \text { Ref. } & \text { Ref. } & \text { Ref. } \\ \text { T 2 } & 634 & 26 & 4.1(2.8-6.0) & 0.92(0.53-1.62) & 0.99(0.56-1.76) & 1.11(0.63-1.95) \\ \text { T 3 } & 426 & 7 & 1.6(0.8-3.4) & 0.38(0.16-0.88) & 0.30(0.13-0.73) & 0.36(0.15-0.86)\end{array}$

$C I$ confidence interval, $D V T$ deep vein thrombosis, $P E$ pulmonary embolism, $S H R$ subdistribution hazard ratio, $T$ tertile.

*Per 100 person-years

†Unadjusted

†Adjusted for age, sex and body mass index

§Competing risk model, adjusted for age, sex and body mass index 